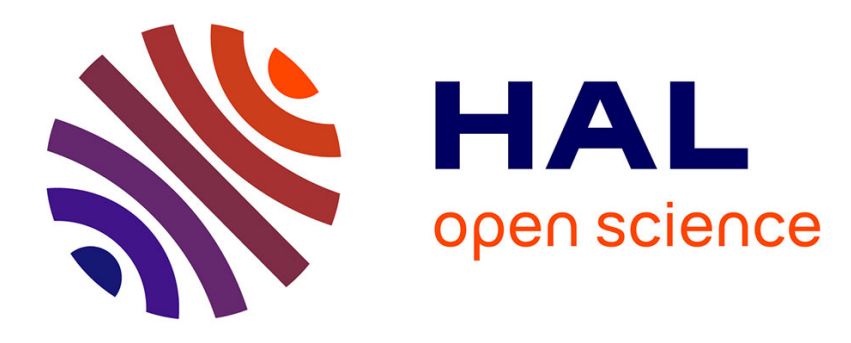

\title{
Structure of Quasicrystals Described by the Statistical Methods
}

\author{
Bartlomiej Kozakowski, Janusz Wolny, Pawel Kuczera
}

\section{To cite this version:}

Bartlomiej Kozakowski, Janusz Wolny, Pawel Kuczera. Structure of Quasicrystals Described by the Statistical Methods. Philosophical Magazine, 2008, 88 (13-15), pp.1921-1927. 10.1080/14786430801974882 . hal-00513875

\section{HAL Id: hal-00513875 \\ https://hal.science/hal-00513875}

Submitted on 1 Sep 2010

HAL is a multi-disciplinary open access archive for the deposit and dissemination of scientific research documents, whether they are published or not. The documents may come from teaching and research institutions in France or abroad, or from public or private research centers.
L'archive ouverte pluridisciplinaire HAL, est destinée au dépôt et à la diffusion de documents scientifiques de niveau recherche, publiés ou non, émanant des établissements d'enseignement et de recherche français ou étrangers, des laboratoires publics ou privés. 


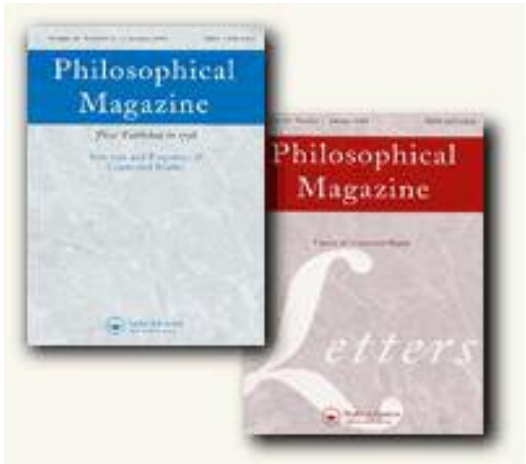

Structure of Quasicrystals Described by the Statistical Methods

\begin{tabular}{|c|c|}
\hline Journal: & Philosophical Magazine \& Philosophical Magazine Letters \\
\hline Manuscript ID: & TPHM-07-Nov-0315.R1 \\
\hline Journal Selection: & Philosophical Magazine \\
\hline $\begin{array}{r}\text { Date Submitted by the } \\
\text { Author: }\end{array}$ & 06-Jan-2008 \\
\hline Complete List of Authors: & $\begin{array}{l}\text { Kozakowski, Bartlomiej; AGH University of Science and Technology, } \\
\text { Faculty of Physics and Applied Computer Science } \\
\text { Wolny, Janusz; AGH University of Science and Technology, Faculty } \\
\text { of Physics and Applied Computer Science } \\
\text { Kuczera, Pawel; AGH University of Science and Technology, Faculty } \\
\text { of Physics and Applied Computer Science }\end{array}$ \\
\hline Keywords: & diffraction, quasicrystals, structure analysis \\
\hline Keywords (user supplied): & diffraction, quasicrystals, structure analysis \\
\hline
\end{tabular}

\section{S ScholarONE" \\ Manuscript Central}




\title{
Structure of Quasicrystals Described by the Statistical Methods
}

\author{
B. Kozakowski, J .Wolny, P. Kuczera \\ Faculty of Physics and Applied Computer Science, AGH - UST, Krakow, Poland
}

\begin{abstract}
The structure of quasicrystals can be described by the statistical approach, which can be used either in higher-dimension or in 3D physical space only. In multidimensional approach whole statistical information is gathered in so called atomic surface. In case of physical approach we use the idea of the average unit cell. These two approaches are linked by an oblique projection. For a full description of a diffraction pattern two projections are required. Statistical methods have been used for the diffraction analysis of the model structures and the structure of real decagonal quasicrystals.
\end{abstract}

\section{Introduction}

Similarly to the modulated structures, quasicrystals can be described either in higherdimensional space (HDS) [1-3] or, alternatively, in only physical space [4]. In the multidimensional analysis, modulated structures become periodic and the atoms get some elongated inner structure called atomic surface (AS), which can be also interpreted as a distribution of all possible structural configurations. One can then say that the atomic surface is the source of the structural information of the real structure but written in the inner space.

Physical space analysis is more difficult as the atoms' arrangement exhibits no period in the real 3D space. It is possible though to enforce the periodicity by overlapping the structure with the reference lattice [4]. This operation would lead to the distribution of atoms' positions with respect to a periodic lattice called an average unit cell (AUC). Fourier transform of the AUC fully describes the diffraction pattern. The AUC can be constructed for every kind of periodic or aperiodic structures; not only for quasicrystals.

These two different approaches, multidimensional analysis and AUC, provide statistical description of the analysed structure and can be successfully used for quasicrystals.

It will be proved in this paper that these two approaches are equivalent and both can be used in diffraction analysis. Theoretical part will be carried out for the Fibonacci chain as the mathematics connected with it is quite brief $[5,6]$. The results will be extended to $2 \mathrm{D}$ quasicrystals: Penrose tiling [7,8] and the decagonal Al-Ni-Co compound [9].

Although the main part of the paper concerns 1D quasicrystal, the conclusions can be straightforwardly applied to the real 2D or 3D structure. It is so, because the structure factor depends on a scalar product of the scattering vector and the positional vector. On the other hand, scalar product can be interpreted as a projection of the structure along the direction of that vector. Projection along one vector is always 1D. It follows that regardless of the dimension of the structure, the diffraction analysis works as if it concerned a 1D structure, which leads to the conclusion that any method tested for 1D can be also applied to 2D and 3D structures.

\section{Oblique projection of atomic surface}

Quasicrystals are not periodic. It is possible however to describe them as modulated structures with an incommensurate modulated wave vector and an AUC. An AUC can be obtained by oblique projection of AS along chosen direction [5-9] (see also fig. 1). The results of this operation are: 
- projected AS gets new interpretation - now it is probability distribution periodically arranged in space, i.e. AUC,

- Fourier transform of the projected AS (AUC) makes it possible to calculate the structure factor only for the main peaks, i.e. for $n \cdot k_{0}$, where $n$ is an integer index; $k_{0}-$ position of the main peak in the physical, reciprocal space,

- reference lattice constant $\lambda$ is related to $k_{0}$ by: $\lambda=2 \pi / k_{0}$,

- the direction of the projection is perpendicular to the direction indicated by the main scattering vector written in HDS.

For an exemplar calculation, we choose the Fibonacci chain $[5,6]$. If the main peak is chosen as $h_{1}=1 ; h_{2}=1$, where $h_{1}, h_{2}$ are $2 \mathrm{D}$ indexes of this peak, then the physical space $k_{0}$ and perpendicular space $k_{0 \perp}$ components are: $k_{0}=k_{\|}(1,1)=k^{\prime} \cdot \tau$ and $k_{0 \perp}=k_{\perp}(1,1)=-k^{\prime} / \tau^{2}$, where $k^{\prime}$ is a constant equal to $2 \pi \tau /(2+\tau)$ and $\tau=2 \cdot \cos (2 \pi / 5)$. In such a case, the lattice constant of AUC is equal to $2 \pi /\left(k^{\prime} \cdot \tau\right)=3-\tau$. The direction of the projection must be perpendicular to the main vector, i.e. $\left(r_{\| \text {proj }}, r_{\perp \text { proj }}\right) \cdot\left(k_{\|}(1,1), k_{\perp}(1,1)\right)=0$, which leads to

$$
r_{\| \text {proj }}=-\left(k_{\perp}(1,1) / k_{\|}(1,1)\right) \cdot r_{\perp \text { proj }}=-r_{\perp \text { proj }} / \tau^{3} .
$$

$r_{\perp \text { proj }}$ is a perpendicular coordinate of an AS and the $r_{\| \text {proj }}$ relates to physical space and describes the coordinates of AUC. The above-mentioned relation bonds these two statistical distributions by means of linear relationship - a projection between $r_{\perp}$ and $r_{\|}$spaces. For the Fibonacci chain, the AS has non-zero values within the range $r_{\perp} \in\left(0, \tau^{2}\right)$. As it follows from the last paragraph, the relation $k_{\perp}(1,1) / k_{\|}(1,1)=\left(-k^{\prime} / \tau^{2}\right) /\left(k^{\prime} \cdot \tau\right)=-1 / \tau^{3}$. When we use this relation on the range $\left(0, \tau^{2}\right)$ we obtain $(-1 / \tau, 0)$ which is the non-zero value range of AUC calculated for the Fibonacci chain.

In such a case, the structure factor reads:

$$
F\left(k_{\|}\right)=\int_{-1 / \tau}^{0} P\left(r_{\|}\right) \exp \left(\mathrm{i} n k_{0} r_{\|}\right) \mathrm{d} r_{\|}
$$

It needs to be emphasized that (1) can describe only a periodic set of main peaks and no satellites can be obtained if the structure is incommensurate. This is a serious disadvantage of (1) as there are much more satellites then main peaks in the diffraction pattern of the Fibonacci chain.

\section{Average unit cell}

Statistical definition of AUC is the distribution of atoms' position with respect to the knots of a periodic reference lattice with a constant $\lambda$ [4]. If the coordinate of $j$-th point relative to the origin is $x_{j}=r_{\| j}$ then with respect to the reference lattice it can be written as $x_{\mathrm{j}}=\alpha_{j} \cdot \lambda+u_{j}$, where $\alpha_{j}$ is an integer giving the number of the knot's position closest to $x_{j}$ and $u_{j}$ is a positive displacement of $x_{j}$ from the $\alpha_{j}$ knot. The lattice constant is defined by the main peak $k_{0}$, i.e. $\lambda=2 \pi / k_{0}$. From above the distances $u_{\mathrm{j}}$ are given by:

$$
u_{j}=x_{j}-\left\|\frac{x_{j}}{\lambda}\right\| \lambda
$$


Here $\|\cdot\|$ is the nearest integer function. We call the series $u_{\mathrm{j}}$ as the displacements sequence of $x_{j}$, induced by the reference periodic lattice with a constant $\lambda$.

For a periodic lattice, structure factor describes only these peaks $k$ which are a multiple of the main peak, i.e. $k=n \cdot k_{0}=n \cdot 2 \pi / \lambda$. For $N$ points in the reference lattice $\lambda$, structure factor reads:

$$
F\left(n k_{0}\right)=\sum_{j=1}^{N} \exp \left(\operatorname{ink} k_{0} x_{j}\right)=\sum_{j=1}^{N} \exp \left(\operatorname{ink} k_{0}\left(\alpha_{j} \lambda+u_{j}\right)\right)=\sum_{j=1}^{N} \exp \left(\mathrm{in} k_{0} u_{j}\right)
$$

Because the set of $u_{j}$ values is limited to $0 \leq u_{j}<\lambda$, the discrete distribution of atoms' positions can be replaced by the continuous probability distribution $P(u)$. In that case, the structure factor is:

$$
F\left(n k_{0}\right)=\int_{0}^{\lambda} P(u) \exp \left(\mathrm{i} n k_{0} u\right) \mathrm{d} u
$$

The structure factor for any harmonics of the scattering vector is an $n$-mod of Fourier transform of the probability distribution of atomic positions in respect to the reference lattice.

\section{Equivalence of two approaches}

Interpretation of equations (1) and (3) is similar. They describe only main peaks and use the probability distributions. If both methods took the same value of $k_{0}$, distributions $P\left(r_{\|}\right)$ and $P(u)$ would have to be identical. This leads to the conclusion that the projection of an AS has the meaning of an AUC given by the statistical approach. Direct confirmation of this thesis can be found in figures $2 a$ and $2 b$. For the Fibonacci chain (fig. 2a) and the Penrose tiling (fig. 2b) numerical calculation of the distribution of atoms in the chosen reference lattice have been conducted (dotted shapes) and compared to the projected borders of the atomic surfaces (thick lines). Projected borders fit perfectly the shape of the numerically calculated distributions.

\section{AUC as a double projection of AS}

Diffraction patterns of 1D quasicrystalline and modulated structures are indexed similarly - by two indexes: $n$ - the multiple of the main peak and $m$ - the number of a satellite. Position of any peak can be written as $k=n \cdot k_{0}+m \cdot q_{0}$, where $q_{0}=k_{0} / \tau$ is the modulation vector. An AUC in the oblique projection approach describes only peaks with $m=0$. In the statistical approach, it is possible to describe all peaks; however; the reference lattice must be applied twice. We define such two lattices, for $k_{0}$ and $q_{0}$ vectors respectively, that for any position $x_{j}$ one can write: $x_{j}=\alpha_{j} \cdot \lambda_{u}+u_{j}$ and $x_{j}=\beta_{j} \cdot \lambda_{v}+v_{j}$, where $\lambda_{u}=2 \pi / k_{0} ; \lambda_{v}=2 \pi / q_{0}$ and the meaning of $\beta_{j}$ is like $\alpha_{j}$ but with respect to the second lattice (both $\alpha_{j}$ and $\beta_{j}$ are integers). For two lattices, structure factor is:

$$
F(k)=\sum_{j=1}^{N} \exp \left(\mathrm{i} k_{0}(n+m / \tau) x_{j}\right)=\sum_{j=1}^{N} \exp \left(\mathrm{i} n k_{0} u_{j}+\mathrm{i} m q_{0} v_{j}\right)
$$

Again, because the sets of values of $u_{j}$ and $v_{j}$ is limited to $0 \leq u_{j}<\lambda_{u}$ i $0 \leq v_{j}<\lambda_{v}$, we can introduce two dimensional probability distribution $P(u, v)$ of atoms' positions.

$$
F(k)=f_{a} \int_{0}^{\lambda_{k}} \int_{0}^{\lambda_{q}} P(u, v) \exp \left(\mathrm{i}\left(n k_{0} u+m q_{0} v\right)\right) \mathrm{d} u \mathrm{~d} v
$$


The $P(u, v)$ distribution fully describes the diffraction pattern and defines the AUC. There is a linear relation between $u$ and $v$ coordinates. The $P(u, v)$ distribution has non-zero values only along a segment bounded by the projected borders of AS. Brief proof of this statement is as follows. $P(u)$ can be obtained by oblique projection, i.e. for every $u$, we have $u=-\left(k_{0 \perp} / k_{0}\right) \cdot r_{\perp}$, where $k_{0 \perp}$ is the perpendicular component of main vector $k_{0}$. The $k_{0}$ vector can be chosen freely, so the same relation must be held for $q_{0}$ vector and $v$ lattice, i.e. $v=-$ $\left(q_{0 \perp} / q_{0}\right) \cdot r_{\perp}$ (Fig. 1). These two oblique projections give the $P(u, v)$ distribution. When $q_{0}=k_{0} / \tau$ then $q_{0 \perp}=-k_{0 \perp} \cdot \tau$ and $v / u=\left(q_{0} / q_{0 \perp}\right)\left(k_{0 \perp} / k_{0}\right)=-\tau^{2}[5,6]$. The last equation leads to the linear relation between the non-zero-value coordinate $u$ and $v: v=-\tau^{2} u$. Relation $v(u)$ and following integration $\int P(u, v) \mathrm{d} v=P(u)$ applied to (5) gives:

$$
F(k)=\int_{0}^{\lambda_{u}} P(u) \exp \left(\mathrm{i}\left(k_{0}(n-m \tau) u\right)\right) \mathrm{d} u
$$

Equation (6) gives full description of the diffraction pattern of the Fibonacci chain and it needs only $P(u)$ distribution to achieve it. $P(u)$ function can be determined for model structures as the oblique projection of AS and for real structures directly from the experimental data. The most important property of $P(u)$ and (6) is that they use only physical information of atoms' arrangement. This equation does not need any additional information which would concern perpendicular space.

\section{Statistical approach of the Penrose tiling}

The perfect Penrose tiling can be described by many structural models. Among them are: rhombus and cluster models. The rhombus model is more flexible - atoms of any type can be put anywhere within the structure unit [7, 8]. In case of the cluster model [11], overlapping rules must be applied. They limit the number of independent atoms.

In order to use equation (6) for the Penrose tiling, it needs to be extended to the second physical dimension. AS of Penrose tiling consists of 4 pentagons. The density of probability distribution is constant within the area of these pentagons.

In the rhombus model there are two structure units: thick and thin rhombus. If a rhombus is decorated only by one atom, the shape of the statistical distribution for one orientation of rhombuses is triangular - as it is shown in the figure 3. To get a full AUC, all 10 orientations must be taken into consideration. Fourier transform, similar to (6), of these triangular distributions allows calculating full diffraction pattern for any decoration of rhombuses. Details for this procedure can be found in [7].

\section{Statistical approach to the structure of Al-Ni-Co}

Statistical approach has been used in order to refine the structure of $\mathrm{Al}_{72} \mathrm{Ni}_{20} \mathrm{Co}_{8}$ compound. The experimental diffraction data was provided by Hiroyuki Takakura. (see also [10]). For our analysis we used 261 averaged and corrected peaks taken from the even diffraction layers. Preliminary calculations were performed on 69 atoms placed in vertices of rhombuses divided four times with obedience to the inflation rules. Additionally, atoms were divided into 19 groups according to the overlapping rules of the cluster model [11]. For most sets of atoms, 6 parameters were refined: displacement $(\Delta x, \Delta y)$ from the starting positions, occupation probability by $\mathrm{Al}$ and $\mathrm{TM}$ atoms and two Debay-Waller parameters. Some parameters of some groups were frozen - not optimized; for instance, atoms put in the vertices of the structure units. Eventually, 110 parameters were refined. We obtained R-factor at the 
level of $10 \%$. Full analysis of obtained results will be published separately. In this paper we would like to indicate only the effectiveness of the statistical approach.

\section{Conclusions}

Full equivalence of statistical methods, i.e. multidimensional one which uses the atomic surface as a source of statistical information and physical-space one which uses the idea of the average unit cell, has been proved. One can use both these methods simultaneously or interchangeably.

To calculate the whole diffraction pattern, the knowledge of $P(u, v)$ distribution of the AUC is required. Such distribution can be obtained in physical space using two reference lattices correlated with incommensurately related wave vectors $k_{0}$ and $q_{0}$. The same distribution can be also obtained by double projection of the atomic surface onto the physical space. Two oblique projections of any point of the atomic surface give two components $u$ and $v$ of the probability distribution. The reverse operation is possible, as well. One can project $u$ and $v$ onto perpendicular space and get the whole information of the atomic surface.

For quasicrystals there is a scaling which leads to some linear relations between $u$ and $v$. Knowing this relation one can reduce $P(u, v)$ to a simpler $P(u)$. This operation essentially simplifies the final expression for the structure factor.

Higher dimensional analysis gives the description of the structure frame in a very simple way. It looses this simplicity when it comes to structure decoration out of Penrose knots. From this point on, it is easier to perform further calculation with the help of an average unit cell which operates only in physical space. Positions of atoms which decorate the rhombuses or clusters modify the structure factor in a way known from the classical crystallography, i.e. by adding to the structure factor amplitudes and phases calculated in relation to the vertices of rhombuses. First, preliminary refinements of the Al-TM structure with the application of the statistical approach are very promising.

\section{Acknowledgements}

The authors would like to acknowledge the National State Committee of Scientific Research for financial support.

\section{References}

1. N.G. de Bruijn, Proc. K. Ned. Akad. Wet. Ser. A 4339 (1981).

2. P. Kramer and R.P. Neri, Acta Crystallogr. A 40580 (1984).

3. V. Elser, Phys. Rev. B 314892 (1985).

4. J. Wolny, Philosophical Magazine A 77395 (1998).

5. B. Kozakowski, J. Wolny, Acta Physica Polonica A 10455 (2003).

6. P. Buczek, L. Sadun, J. Wolny, Acta Physica Polonica B 36919 (2005).

7. B. Kozakowski and J. Wolny, Structure factor for decorated Penrose tiling, Available online at: http://arxiv.org/ftp/cond-mat/papers/0503/0503464.pdf (2005).

8. B. Kozakowski, J. Wolny Philosophical Magazine 86549 (2006).

9. W. Steurer, T. Haibach, Acta Cryst. A 5548 (1999), 
10. H. Takakura, A. Yamamoto, A.P. Tsai, Acta Cryst. A 57576 (2001).

11. A. Dąbrowska, B. Kozakowski, J. Wolny, Acta Cryst. A 61350 (2005).

\section{Figure captions:}

Fig. 1. Double oblique projection of the atomic surface onto physical space gives the probability distribution $P(u, v)$ and along with it the average unit cell. Two vectors $\mathbf{K}$ and $\mathbf{Q}$ are the multidimensional equivalents of the physical space wave vectors $\mathbf{k}$ and $\mathbf{q}$. The $u$ coordinate of the probability distribution is an oblique projection of the $r_{\perp}$ coordinate of the atomic surface. The direction of the projection is perpendicular to the wave vector $\mathbf{K}$. The same procedure applied to $\mathbf{Q}$ wave vector leads to the $v$ coordinate of the probability distribution.

Fig. 2. (to the left) Probability distribution of atomic positions of the Fibonacci chain with respect to the reference lattice $\left(\lambda=1+1 / \tau^{2} \approx 1.38\right)$ was numerically calculated for about 1000 atomic positions. The obtained AUC is identical (within the statistical accuracy) to the projected atomic surface (solid line).

(to the right) The same as above but for the Penrose tiling (10 000 points). Also in this case, the calculated probability distribution and the obtained oblique projection (thick lines) of atomic surface are the same.

Fig. 3. After projection of two pentagons of the atomic surface onto physical space (shown in the figure) one obtains the average unit cell of the Penrose tiling. The marked triangles of the AUC describe the distribution of positions of the vertices of the thick (to the left) and thin (to the right) rhombuses. The positions were calculated for a chosen orientation. 


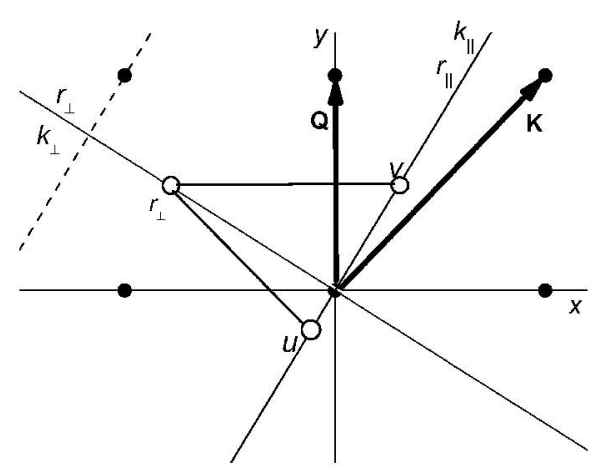

Figure 1

$209 \times 297 \mathrm{~mm}(600 \times 600$ DPI $)$ 


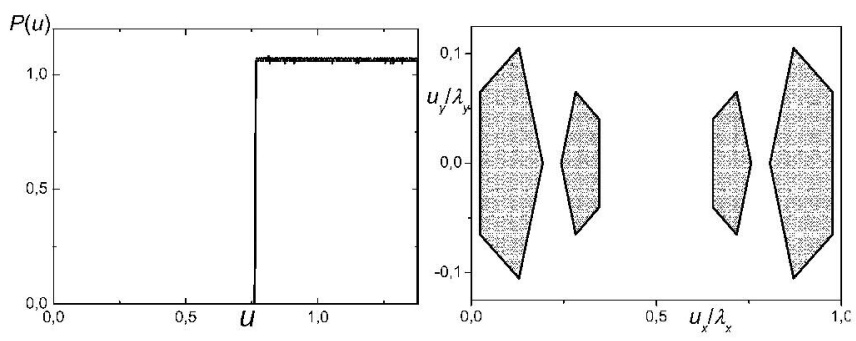

Figure 2 $209 \times 297 \mathrm{~mm}(600 \times 600$ DPI $)$ 


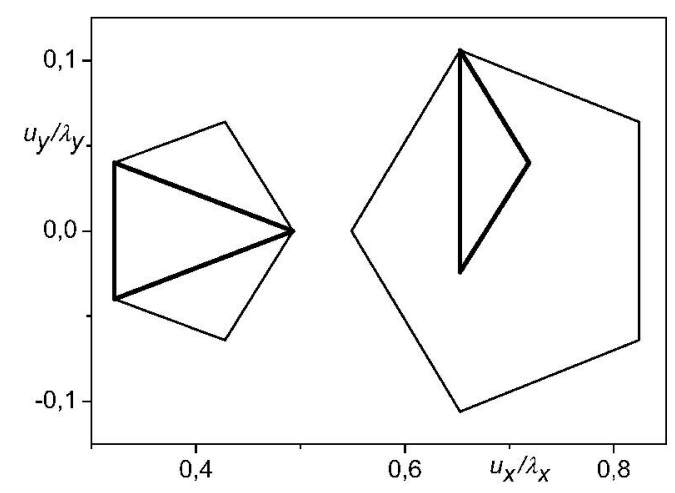

Figure 3

$209 \times 297 \mathrm{~mm}(600 \times 600$ DPI $)$ 